\title{
Síndrome do nevo de Becker
}

\author{
Becker nevus syndrome
}

\author{
Fabiane Eiras Cosendey ${ }^{1}$ \\ Gabriela Alice Bernhard ${ }^{3}$ \\ David Rubem Azulay ${ }^{5}$
}

\author{
Nayibe Solano Martinez ${ }^{2}$ \\ Maria Fernada Reis Gavazzoni Dias ${ }^{4}$
}

Resumo: A síndrome do nevo de Becker é considerada um fenótipo caracterizado pela presença fundamental do nevo de Becker associado à hipoplasia mamária unilateral ou a outras alterações cutâneas, esqueléticas e/ou musculares. Geralmente, surge ao nascimento, aumenta significativamente na adolescência e é uma das síndromes que constituem a síndrome do nevo epidérmico. O artigo relata o primeiro caso da literatura brasileira da síndrome do nevo de Becker em uma paciente de 14 anos com associação de nevo de Becker, hipoplasia mamária unilateral e escoliose.

Palavras-chave: Escoliose; Hamartoma; Hipertricose; Nevo

\begin{abstract}
Becker nevus syndrome is a phenotype characterized by the fundamental presence of Becker's nevus with ipsilateral hypoplasia of the breast or other skin, skeletal and/or muscular disorders. This syndrome generally originates at birth, intensifies significantly in adolescence and is one of the syndromes that constitute epidermal nevus syndrome. To the best of our knowledge, this is the first case published in the Brazilian literature of Becker nevus syndrome associated with Becker's nevus, ipsilateral breast hypoplasia and scoliosis in a 14-year-old girl.

Keywords: Hamartoma; Hypertrichosis; Nevus; Scoliosis
\end{abstract}

\section{INTRODUÇÃO}

O nevo de Becker (NB) é um hamartoma cutâneo caracterizado por hiperpigmentação circunscrita com hipertricose, que foi descrito pela primeira vez por Becker, em 1949.,

Muitos autores, no passado, observaram a associação do NB com outras anomalias, como hipoplasia de mama ou escoliose. ${ }^{1}$ Porém, foi somente em 1995 que o termo síndrome do nevo epidérmico piloso ou síndrome do nevo de Becker foi descrito pela primeira vez, por Happle, como uma associação do NB com hipoplasia mamária unilateral, alterações musculares, cutâneas e/ou esqueléticas. ${ }^{1}$ Essa entidade, descrita recentemente, faz parte da síndrome do nevo epidérmico.

\section{RELATO DO CASO}

Paciente feminina, 14 anos, solteira, moradora de Realengo, refere que, desde o nascimento, apresentava mancha de pequeno tamanho e levemente acastanhada na mama direita. Relata que, na adolescência, a lesão estendeu-se para dorso e membro inferior direito, com aumento de pêlo no local. Nega hirsutismo, irregularidade menstrual e história familial. Ao exame dermatológico, apresentava mácula hipercrômica de bordas bem definidas e irregulares na região escapular direita, que se estendia para ombro e região anterior do tórax, e mácula hipercrômica com hipertricose em membro inferior direito ipsilateral (Figuras 1 e $2 \mathrm{~A}$ ).

\footnotetext{
Recebido em 07.05.2009.

Aprovado pelo Conselho Consultivo e aceito para publicação em 27.11.2009.

* Trabalho realizado no Instituto de Dermatologia Professor Rubem David Azulay da Santa Casa da Misericórdia do Rio de Janeiro - Rio de Janeiro (RJ), Brasil. Conflito de interesse: Nenhum / Conflict of interest: None

Suporte financeiro: Nenhum / Financial funding: None

Pós-graduanda de Dermatologia do Instituto de Dermatologia Professor Rubem David Azulay - Santa Casa de Misericórdia do Rio de Janeiro; residência em Clínica Médica no Hospital Central da Polícia Militar do Rio de Janeiro - Rio de Janeiro (RJ), Brasil.

Pós-graduanda de Dermatologia do Instituto de Dermatologia Professor Rubem David Azulay - Santa Casa de Misericórdia do Rio de Janeiro - Rio de Janeiro (RJ), Brasil.

Pós-graduanda de Dermatologia do Instituto de Dermatologia Professor Rubem David Azulay - Santa Casa de Misericórdia do Rio de Janeiro - Rio de Janeiro (RJ), Brasil

Professora de Dermatologia do Instituto de Dermatologia Professor Rubem David Azulay - Santa Casa de Misericórdia do Rio de Janeiro; doutora e mestre em Dermatologia na Universidade Federal do Rio de Janeiro (UFRJ) - Rio de Janeiro (RJ), Brasil.

Chefe de Serviço do Instituto de Dermatologia Professor Rubem David Azulay - Santa Casa de Misericórdia do Rio de Janeiro; professor titular da Pontifícia Universidade Católica (PUC/RJ); professor regente de Dermatologia da Fundação Técnico-Educacional Souza Marques; professor assistente da Universidade Federal do Rio de Janeiro (UFRJ) - Rio de Janeiro (RJ), Brasil.
} 

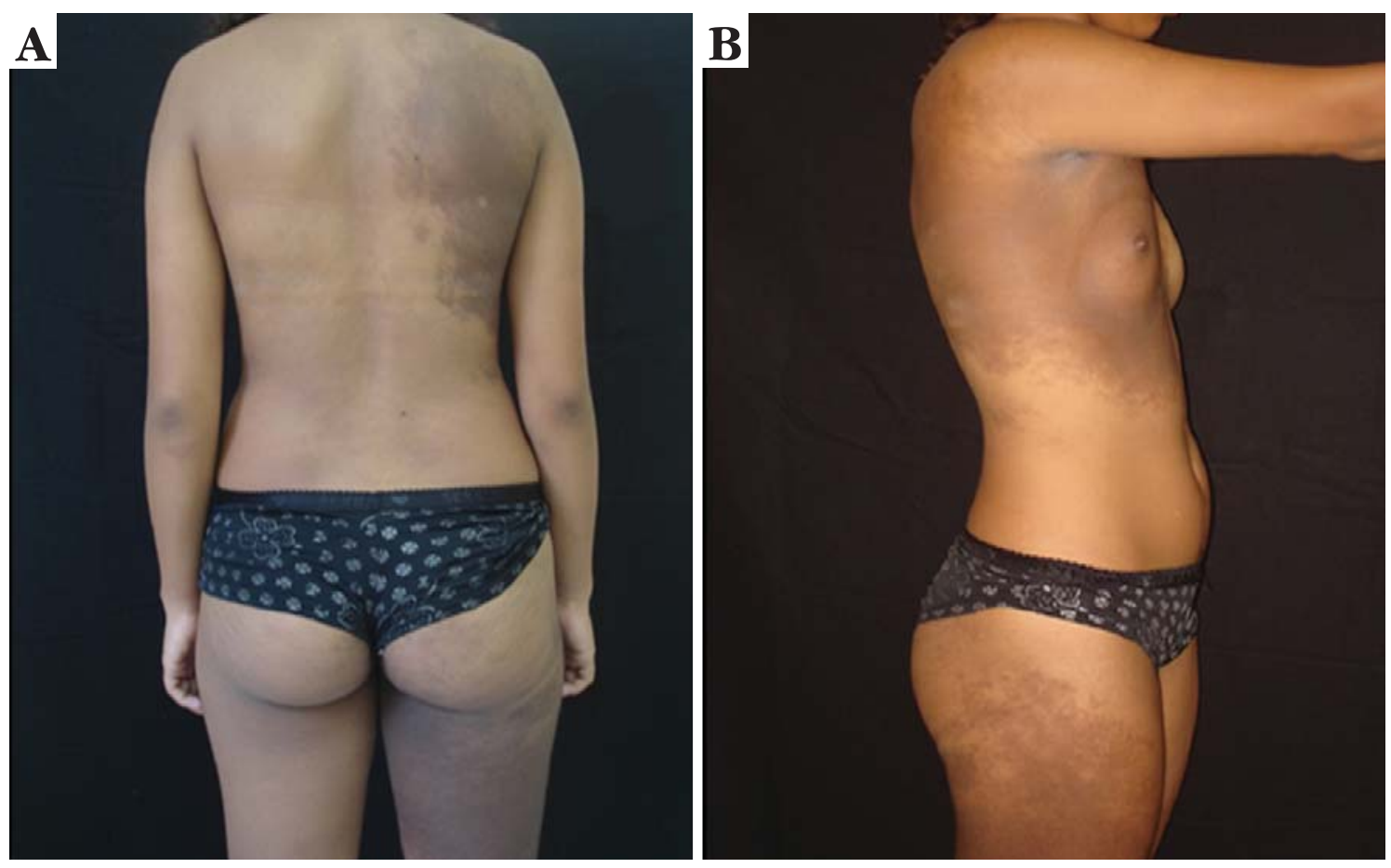

Figura 1: A e B. Mácula hipercrômica de bordas bem definidas e irregulares na região escapular direita que se estende para ombro e região anterior do tórax na SNB

Ao exame clínico, apresentava hipogenesia mamária direita, escoliose e exame ginecológico normal (Figura 2B). O exame histopatológico da mácula evidenciou acantose moderada, hiperpigmentação da camada basal com grânulos grosseiros de melanina, presença de melanófagos na derme papilar e hiperplasia da musculatura eretora do pêlo, compatível com nevo de Becker (Figura 3). Foram realizados os seguintes exames laboratoriais: hemograma, função hepática, função renal, $17 \mathrm{OH}$ Progesterona, LH, FSH, Prolactina, $\mathrm{T}_{4}$ livre e TSH, que foram normais. Nos exames de imagem, evidenciaram-se: radiografia de coluna, com presença de escoliose, e ultrassonografia de mama, com espessamento tecidual fibroglandular da mama esquerda e mama direita hipotrófica.

\section{DISCUSSÃO}

O NB é um hamartoma hiperpigmentado com hipertricose, de bordas bem definidas, que pode ocorrer em qualquer parte do corpo, principalmente, no tronco e nos membros superiores. ${ }^{1,2,3}$ Pode ocorrer regressão espontânea da lesão. Em 1984, Person e Longcope detectaram um aumento dos níveis de receptores de androgênio no nevo de Becker. Estudos posteriores confirmaram esse aumento, considerando a hipótese da hiper-responsividade hormonal desse hamartoma cutâneo. Na literatura, há relatos de alguns pacientes com lesões acneiformes no nevo de Becker e tem sido proposta a hipótese de essa lesão ser mediada por androgênio. Por ser uma
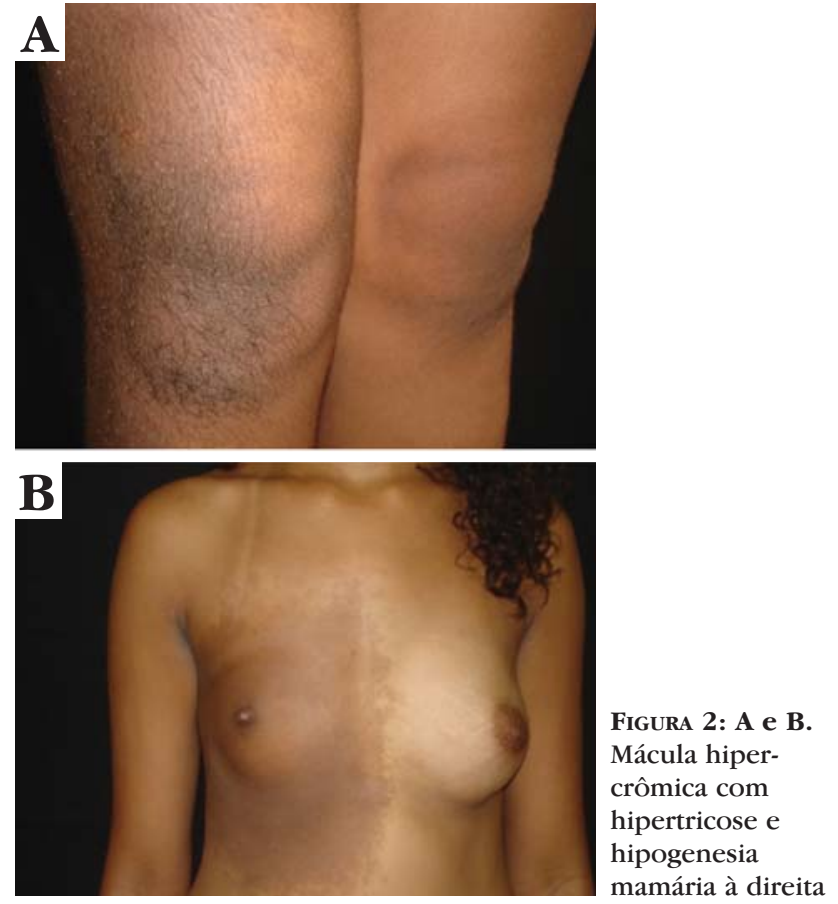

enfermidade dependente de androgênio, é muito mais comum em homens (5:1) e durante a adolescência. ${ }^{1,4}$

$\mathrm{Na}$ histopatologia dessa síndrome, observa-se, na epiderme, acantose e hiperpigmentação da camada basal, enquanto que, na derme, evidencia-se a hiperplasia da musculatura eretora do pelo com melanófagos, como visto na paciente em questão. ${ }^{1,2,4}$ 

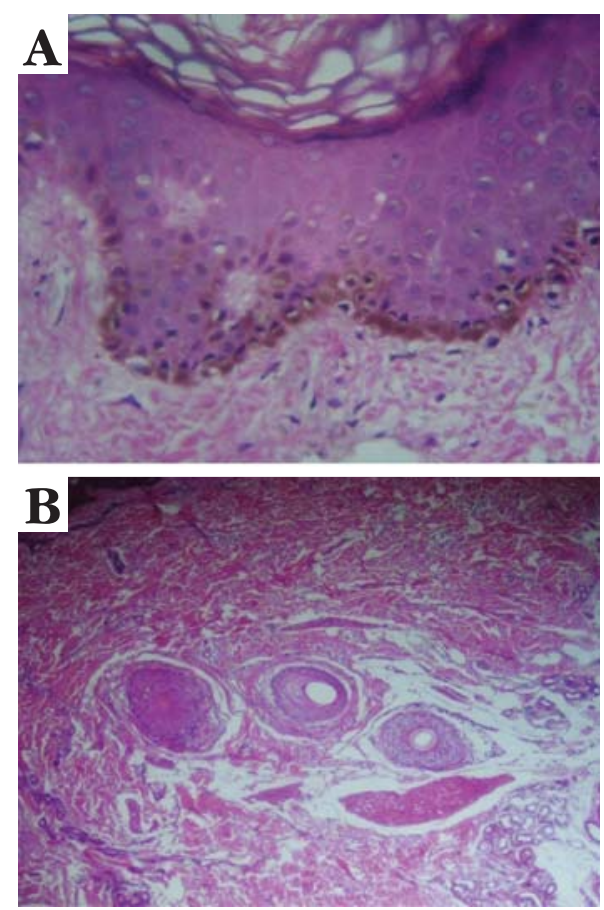

Figura 3:

A. Hiperpigmentação da camada basal com grânulos grosseiros de melanina; B. Hiperplasia da musculatura lisa eretora do pelo

$\mathrm{O} \mathrm{NB}$, apesar da hipercromia, não pertence ao grupo das lesões melanocíticas. Ele é considerado uma forma especial do nevo epidérmico (NE), como o nevo epidérmico verrucoso, o nevo sebáceo de Jadassohn, o nevo comedônico, o nevo écrino, o nevo apócrino e o nevo espongiforme branco, que são assim denominados de acordo com a estrutura preponderante (Quadro 1). ${ }^{1,5}$

Conceitualmente, o NE é considerado uma proliferação hamartomatosa desenvolvida do ectoderma embriogênico, caracterizada por hiperplasia de estruturas epidérmicas como queratinócitos, glândulas apócrinas, écrinas, folículo

Quadro 1: Classificação do nevo epidérmico

\begin{tabular}{|ll|}
\hline Variantes & Estrutura predominante \\
Nevo epidérmico verrucoso & Superfície epidérmica \\
Nevo comedônico & Folículo piloso \\
Nevo écrino & Glândula sudorípara écrina \\
Nevo apócrino & Glândula sudorípara apócrina \\
Nevo de Becker & Superfície epidérmica, folículo \\
piloso, musculatura lisa \\
Nevo espongiforme branco
\end{tabular}

piloso e glândula sebácea. ${ }^{5}$

Em 1968, Solomon et al. descreveram a síndrome do nevo epidérmico (SNE), que é constituída pela associação do NE, em geral, mais extenso, com diversas anormalidades cutâneas, oculares, do sistema nervoso central (SNC), esqueléticas, que têm origem no ectoderma e podem, mais raramente, ser acompanhadas de alterações urogenitais e cardiovasculares que derivam a partir do mesoderma embriogênico. ${ }^{5,6,7}$

$\mathrm{O}$ aspecto cutâneo do NE depende, em parte, do tipo predominante de célula envolvida, da idade do paciente, do local do corpo acometido e do grau de diferenciação celular. O NE segue as linhas de Blaschko. A associação do NE com anormalidades do SNC foi descrita, pela primeira vez, por Schimmelpenning e, posteriormente, por Feuerstein e Mims. ${ }^{3}$ Quanto maior o número de lesões extracutâneas e quanto mais disseminado o NE, maior a probabilidade de envolvimento do SNC e de outras manifestações sistêmicas, o que pode ser um reflexo da gravidade da herança biológica causada por mutação durante o seu desenvolvimento. ${ }^{5,6,8}$ Cerca de $8 \%$ dos pacientes com NEs apresentam acometimento sistêmico. Além do NE, podem ocorrer outras alterações cutâneas na SNE, como manchas café-aulait, nevo lentiginoso salpicado, múltiplos nevos melanocíticos e malformações vasculares (facomatose pigmentovascular). ${ }^{5}$ Fazem parte de um conceito ainda maior da SNE as seguintes entidades descritas por Happle: síndrome de Proteus, síndrome do nevo sebáceo, síndrome do nevo de Becker, facomatose pigmentoceratótica, síndrome Child, síndrome do nevo comedônico (Quadro 2).$^{5,6,8}$

Em 1995, Happle descreveu, pela primeira vez, a síndrome do nevo de Becker (SNB) ou síndrome do nevo epidérmico piloso. A presença do NB é fundamental para caracterizar a síndrome, porém, é necessária a sua associação com hipoplasia mamária ou com outras alterações cutâneas, musculares e/ou esqueléticas (Quadro 3). ${ }^{1,2}$ Em geral, essas anomalias envolvem o mesmo lado do corpo, mas podem ser bilaterais. Hipogenesia mamária, escoliose ou quaisquer outros achados não são suficientes, isoladamente, para o diagnóstico da síndrome. ${ }^{1}$

A SNB também é uma anomalia dependente de androgênio, assim como o NB, sendo, por isso, em geral, mais observada na adolescência, pelo aumento da hiperpigmentação do nevo e do pêlo. ${ }^{1,2,3,4,5} \mathrm{~A}$ proporção de mulheres afetadas é igual à de homens, apesar de a síndrome ser mais facilmente diagnosticada em mulheres, pela presença mais visível de hipoplasia mamária. ${ }^{1,2}$

Acredita-se que muitos dos achados associados à síndrome sejam decorrentes do aumento do 
QUADRo 2: Classificação da síndrome do nevo epidérmico

\begin{tabular}{|c|c|}
\hline Síndrome de Proteus & $\begin{array}{l}\text { Nevo epidérmico verrucoso-ceratótico linear, gigantismo parcial das mãos } \\
\text { e pés, hiperplasia plantar cerebriforme, hemangiomas, lipomas, } \\
\text { lipo-hipoplasia, hipoplasia dérmica focal, macrocefalia, hiperostose, } \\
\text { hipoplasia muscular, hipertrofia de ossos longos }\end{array}$ \\
\hline $\begin{array}{l}\text { Síndrome do nevo sebáceo } \\
\text { (Schimmelpenning) }\end{array}$ & $\begin{array}{l}\text { Nevo sebáceo associado a anormalidades cerebrais, coloboma e } \\
\text { lipodermoide da conjuntiva }\end{array}$ \\
\hline Síndrome do nevo comedônico & Associada à catarata, à escoliose e a anormalidades neurológicas \\
\hline $\begin{array}{l}\text { Síndrome Child (hemidisplasia congênita } \\
\text { com eritrodermia ictiosiforme e } \\
\text { defeitos dos membros) }\end{array}$ & $\begin{array}{l}\text { Nevos epidérmicos inflamatórios unilaterais e hipoplasia ou defeitos dos } \\
\text { membros ipsilaterais. Pode haver comprometimento dos sistemas músculo } \\
\text {-esquelético, cardiovascular ou nervoso central }\end{array}$ \\
\hline Facomatose pigmentoceratótica & $\begin{array}{l}\text { Associação do nevo sebáceo organoide, nevo lentiginoso salpicado, } \\
\text { raquitismo hipofosfatêmico }\end{array}$ \\
\hline Síndrome do nevo de Becker & $\begin{array}{l}\text { Nevo de Becker, hipoplasia ipsilateral de mama e defeitos esqueléticos, } \\
\text { musculares e/ou cutâneos }\end{array}$ \\
\hline
\end{tabular}

QUADro 3: Achados clínicos da síndrome do nevo de Becker

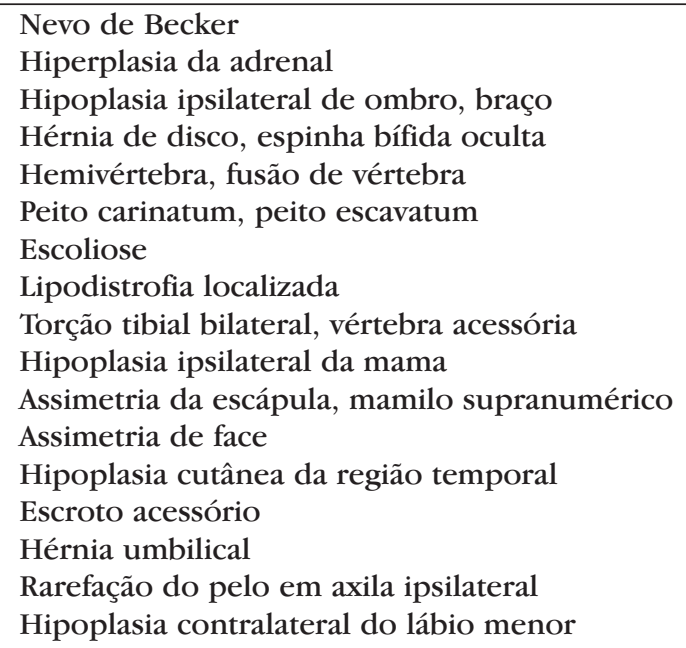

número de receptores de androgênio em determinadas áreas. $O$ androgênio é um dos hormônios responsáveis pelo crescimento do pelo, do desenvolvimento ósseo e da massa muscular. Person e Nird et al. mediram os receptores de androgênio na lesão do NB e na pele normal de um paciente com NB e encontraram um aumento desses receptores na lesão. Eles propuseram que o NB poderia ser considerado um componente do espectro da síndrome de hipersensibilidade do receptor de androgênio.' A patogênese da hipoplasia da mama ainda não é conhecida, mas é possível que seja a mesma que a do NB, sendo que, na mama, os níveis dos receptores de androgênio podem contrabalançar o efeito do estrogênio no crescimento da mama normal, resultando em uma mama hipoplásica.

A espironolactona é um antiandrogênio usado na acne, no hirsutismo e na alopecia androgenética. O mecanismo não é muito bem conhecido, mas acreditase que possa resultar da retroalimentação negativa dos receptores de androgênio e assim, então, melhorar a hipoplasia. Neste estudo, observou-se um aumento da mama com hipoplasia após uso de espironolactona 50 $\mathrm{mg} / \mathrm{dia} .{ }^{9}$ Esse achado corrobora a hipótese do aumento dos receptores de androgênio no NB e na mama hipoplásica. Entretanto, não foi possível observar esse achado na paciente em questão, já que ela preferiu submeter-se à cirurgia de prótese de mama.

$\mathrm{Na}$ maioria dos casos, a síndrome ocorre esporadicamente e raramente mostra associação familial. Indivíduos heterozigóticos seriam fenotipicamente normais e o alelo responsável seria, então, transmitido através de muitas gerações. Estudos genéticos sugerem que o nevo e a associação de anomalias seriam somente originados quando uma recombinação somática ocorresse no estágio precoce da embriogênese, resultando em uma população de células homozigóticas. ${ }^{1,6}$ Porém, a base genética dessa síndrome ainda não foi muito bem esclarecida. A SNB tem achados clínicos muito sutis e é provável que muitos casos tenham passado despercebidos. 


\section{REFERÊNCIAS}

1. Happle R, Koopman RJJ. Becker nevus syndrome. Am J Med Genetics. 1997;68:357-61.

2. Alfaro A, Torrelo A, Hernández A, Zambrano A, Happle R. Síndrome del nevo de Becker. Actas Dermosifiliogr. 2007;98:624-6.

3. Ferreira MJ, Bajanca R, Fiadeiro T. Congenital melanosis and hypertrichosis in a bilateral distribution. Pediatri Dermatol. 1998;15:290-2.

4. Santos-Juanes J, Galache C, Curto JR, Carrasco MP, Ribas A, Sánchez del Río J. Acneiform lesions in Becker's nevus and breast hypoplasia. Int J Dermatol. 2002;41:699-700.

5. Thomas VD, Swanson NA, Lee KK. Benignan epithelial tumors, hamartomas and hyperplasias. In: Wolff $\mathrm{K}$, Goldsmith LA, Katz SI, Gilchrest BA, Paller AS, Lefell D, editors. Fitzpatrick's Dermatology in General Medicine; 7th ed. New York: McGraw-Hill; 2008. p.1057-62.

6. Sugarman JL. Epidermal nevus syndromes. Semin Cutan Med Surg. 2007;26:221-30.
7. Noronha L, Neto JF, Nones RB, Taniguchi K. Nevo epidérmico: análise clínica e histológica de seis casos. An Bras Dermatol.1999;74:259-62.

8. Vidaurri-de la Cruz H, Tamayo-Sánchez L, DuránMcKinster C, de la Luz Orozco-Covarrubias M, RuizMaldonado R. Epidermal nevus syndromes: Clinical findings in 35 patients. Pediatr Dermatol. 2004;21:432-9.

9. Hoon Jung J, Chan Kim Y, Joon Park H, Woo Cinn Y. Becker's nevus ipsilateral breast hypoplasia: improvement with Spironolactona. J Dermatol. 2003;30:154-156.

ENDEREÇO PARA CORRESPONDÊNCIA / MAILING ADDRESS:

Fabiane Eiras Cosendey

Rua Mariz e Barros, 71 - Ap. 902 - Icaraí

24220120 Niteró $i$ - RJ

Tel.: 212612162078950462

E-mail: fabianecosendey@yaboo.com.br

Como citar este artigo/How to cite this article: Cosendey FE, Martinez NS, Bernhard GA, Dias MFRG, Azulay DR. Síndrome do nevo de Becker. An Bras Dermatol. 2010;85(3):379-84. 